\title{
MODELING THE ROBO-ADVISOR ECOSYSTEM: INSIGHTS FROM A SIMULATION STUDY
}

\author{
Thomas L. Ngo-Ye, Alabama State University,tngoye@alasu.edu \\ Jae J. Choi, Pittsburg State University,jchoi@pittstate.edu \\ Maeve Cummings, Pittsburg State University, cummings@pittstate.edu
}

\begin{abstract}
Many FinTech (Financial Technologies) have evolved to disrupt the whole industry. Robo-Advisors are one of the recent incarnations of FinTech, attracting more and more attention by pitching a variety of potential advantages including cost saving and transparency in the financial service industry. Yet it is still too soon to tell whether what we are hearing is just hype or not. Anecdotal evidence and observations are contradictory and largely divided as to whether Robo-Advisors will join other disruptive FinTechs in the foreseeable future. This paper posits trust as one of the main factors affecting Robo-Advisors' adoption and eventual success. It documents the development of an agent-based simulation model to examine the impact of key elements in the trust building processes. The aim is to identify the decision making process and environmental conditions under which Robo-Advisors can be successfully implemented. A design science approach is adopted due to the dynamic nature and complexity of trust building mechanisms. This study reports on the second stage of a comprehensive study which aims to examine broad strategies for the implementation and deployment of Robo-Advisors.
\end{abstract}

Keywords: FinTech, Robo-Advisors, IT adoption, Diffusion, Trust, Agent-based modeling

\section{INTRODUCTION}

FinTech continues to be a topic that is of interest to the finance industry as well as to the IT (Information Technology) community. It has been a vehicle for disruptive innovation in the financial industry, which has a long history of successfully adopting innovative technologies ranging from automated teller machines to online banking in recent years. Robo-Advisors emerged as the latest FinTech after the 2008 financial crisis, in the wake of diminishing trust in traditional financial institutions (Salo, 2017). It offers investment advice using an algorithm-based process without human interaction (Gold \& Kursh, 2017). Over the last decade, Robo-Advisors have become an increasingly significant phenomenon. Their adoption rate is predicted to skyrocket, reaching 5.6\% in 2020 from a mere 0.5\% in 2015 (Epperson, Hedges, Singh, \& Gabel, 2015). This indicates that up to two trillion dollars are expected to be managed under Robo-Advisors by 2020. If the prediction is correct, Robo-Advisors might well be poised to become prominent disruptors in the ETF (Exchange-traded Fund) market, surpassing the historical adoption speed of market disruption in the finance industry (Epperson, Hedges, Singh, \& Gabel, 2015).

Conversely, much contrasting anecdotal evidence has since emerged. One survey indicates that the percentage of respondents who are highly willing to try Robo-Advisors dropped in 2017 compared with the previous year (Reuba, 2017). Perhaps a more alarming result is that financial advisors do not find that Robo-Advisors have a strong effect on their businesses. Echoing this view, Nanalyze, an investor information service group, expressed disappointment with Robo-Advisors, stating that the embedded technology does not live up to the hype and that Robo-Advisors are more about process automation than AI (artificial intelligence) or deep learning (Nanalyze, 2016).

The conflicting opinions on Robo-Advisors stem partially from the fact that there are multiple generations of Robo-Advisors. Each generation is equipped with a vastly different set of features. Accordingly, there has been much confusion about the expected features and services that Robo-Advisors have to offer. Moulliet, Stolzenbach, Majonek, and Völker (2016) proposed four different generations of Robo-Advisors. The first generation offers basic Web questionnaire-based customization. Customers receive customized product proposals or portfolio allocations based on the information collected through Web questionnaires. Although the Web is used as a medium of communication, human advisors play the pivotal role behind the scenes. The second generation of Robo-Advisors saw technology being used for more than simple communication. This time a set of dedicated tools was available to help financial advisors manage risk-based portfolio allocation and rebalancing. The procedure was semi-automatic as human financial advisors still oversee the investment algorithm and define rule sets. The third 


\section{Issues in Information Systems \\ Volume 19, Issue 1, pp. 128-138, 2018}

generation of Robo-Advisors finally implemented embedded algorithms and predefined rule sets, limiting human involvement to minimum levels; the final oversight and approval. About $80 \%$ of European and American Robo-Advisors fall into the thirdgeneration category and the remainder are equipped with first- or second-generation features. The latest generation promises fully automated investing utilizing self-learning algorithms. A shift between different asset classes is possible with the help of artificial intelligence (AI). Such fourth generation Robo-Advisors are just beginning to emerge.

It is not surprising that the primary benefit of Robo-Advisors is proclaimed to be cost saving due to a high degree of automation (Epperson, Hedges, Singh, \& Gabel, 2015; Salo, 2017) considering the prevalence of third generation tools in the market. Ironically, this hampers the prospect of Robo-Advisors' role as a disruptive innovation as the market expects more than just cost cutting. One survey indicates that one of the critical factors when selecting a Robo-Advisor service is investment expertise (Epperson, Hedges, Singh, \& Gabel, 2015). A subsequent study noted that transparency through a consolidated user interface is another key dimension to be considered (Salo, 2017). Within the context of Robo-Advisors, transparency is part of the feature that allows users to understand the relevant circumstances as a whole without a financial expert's interpretation or advice. Our view is that both investment expertise and transparency are key factors affecting the trustworthiness of Robo-Advisors. Prior studies have found that trust plays a major role in the adoption of digitalization technology in service industries in general (Reichheld \& Schefter, 2000). The trust issue is even more pronounced in the financial industry.

The purpose of study is to model the Robo-Advisor Ecosystem using a simulation approach. Our current study explores the environmental conditions and design decisions that ensure the successful deployment, adoption, and evolution of RoboAdvisors into a major disruptive innovation. Our main focus is the dynamic and complex trust building mechanisms involved in the adoption of Robo-Advisors. Although trust has been identified as a key component that shapes the nature of digitalization technologies, the majority of the previous studies focused on the identification of trust antecedents from a static perspective, and did not address the inherently dynamic trust lifecycle. The emergence of newly available features in the fourth generation of Robo-Advisors makes this investigation even more challenging. In order to study the effect of design and other environmental conditions on the Robo-Advisor market, a dynamic strategy is called for, wherein individual users and RoboAdvisors can be modeled. This research constructs an agent-based model that encompasses Robo-Advisor providers and customers.

Adopting the design science research approach suggested in (Gregor \& Hevner, 2013) and (Peffers, Tuunanen, Rothenberger, \& Chatterjee, 2008), which draw from ideas outlined in (Hevner, March, Park, \& Ram, 2004), this manuscript reports on the second stage of the development of a dynamic Robo-Advisor trust model that captures the effects of alternative strategies involved in the implementation of a Robo-Advisor. The initial stages and overview were reported in our previous study (Lee, Choi, Ngo-Ye, \& Cummings, 2018).

\section{TRUST AND ITS ANTECEDENTS}

Trust has assumed an increasingly important role as e-commerce matures (Suh \& Han, 2003). Fear and anxiety are inevitable when transactions are conducted with remotely located and unknown partners. The perceived risk coupled with trust in online transactions play a key role in people's willingness to engage in online transactions. Generally, trust is considered to be a critical precondition for the adoption of electronic services involving more than simple online transactions (Beldad, De Jong, \& Steehouder, 2010).

Initial trust refers to trust in an unfamiliar trustee, a relationship in which the actors do not yet have credible, meaningful information about each other, and have not yet developed mutual effective bonds (Bigley \& Pearce, 1998). In the case of ecommerce, trust is transaction-based and shaped by individual transactions. Hence, studies in e-commerce have focused primarily on how to establish initial trust during the period when a consumer visits and explores a vendor's Web site for the first time (McKnight, Choudhury, \& Kacmar, 2002). Unlike the case of e-commerce, trust in Robo-Advisors and other FinTech services require a longer-term orientation. This type of trust is characterized as ongoing trust and formed by a calculus-based trust rather than perceptions or initial impressions (Lewicki \& Bunker, 1995). Another basis of ongoing trust is knowledgebased trust, which develops over time with the accumulation of trust-relevant knowledge resulting from experience with the transaction partner (Holmes, 1991). Ongoing trust in the context of Robo-Advisors represents the customer's trust built upon knowledge and calculation from outcomes accumulated over a long-term investment advising relationship. Key stages in the ongoing trust lifecycle involve trust violation and restoration. Trust violations result in a cognitive appraisal and an emotional reaction, leading to lower knowledge- and calculus-based trust (Lewicki \& Bunker, 1996). The nature, magnitude and severity 
of the offense triggers an assessment on the part of the victim. The accumulated trust gained through the prior relationship is eroded and leads to decreased knowledge-based trust. Reconciliation can play a role in restoring broken or degraded trust. A process view of trust violation and restoration is depicted in Figure 1, as adapted from (Lewicki \& Bunker, 1996).

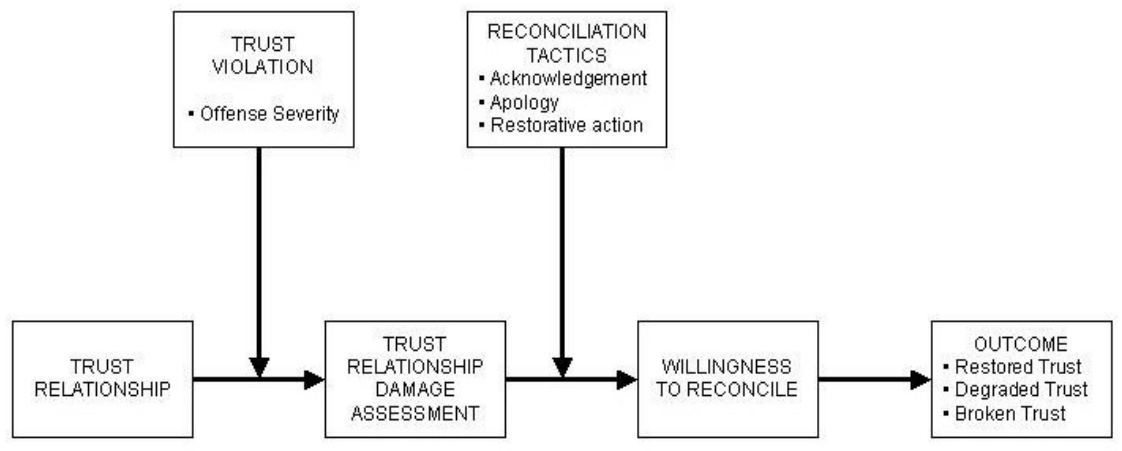

Figure 1. Trust Violation and the Reconciliation Process

\section{SECURITY AND TRUST}

A number of factors have been identified as contributing to trust. One critical trust antecedent in FinTech tools concerns security and privacy. Users view security as a vital criterion in the assessment of trustworthiness (Aiken \& Bousch, 2006). The importance of security and privacy is more pronounced in the financial service industry because financial information is one of the main targets of fraud (Roca, Carcia, \& De La Vega, 2009). Security breaches will only worsen as consumers need and gain more access to data and applications. A variety of security tools and techniques are available to implement the five categories of security controls; authentication, non-repudiation, privacy, confidentiality, and data integrity controls (Ray, Ow, $\& \mathrm{Kim}, 2011)$. When these tools and techniques are successfully implemented perceived security improves. Perceived security refers to "the subjective probability with which consumers believe that their personal information will not be viewed, stored, or manipulated during transit and storage by inappropriate parties in a manner consistent with their confidence expectations" (Flavian \& Guinaliu, 2006). Perceived security rather than security artifacts is the primary factor affecting the formation of trust because users generally lack the understanding of security technologies (Chellappa \& Pavlou, 2002) and security controls are not easily comprehended (Ray, Ow, \& Kim, 2011). Choi and Nazareth (2014) adapted (Lewicki \& Tomlinson, 2003)'s framework for the rebuilding of interpersonal trust after security breaches in electronic service industries as follows:

"In the security and e-commerce context, the process is expected to be a little different. The vendor may not be aware that a security breach has occurred. Likewise, the consumer may also be in the dark about this breach. However, if an adverse incident occurs, then the initiation will likely originate from the consumer-the victim in this case. The extent to which the vendor acknowledges the incident, apologizes, and offers appropriate restorative action, will shape the extent that the consumer is willing to reconcile. Other factors at this stage include the need for maintaining an ongoing relationship with this vendor, and the availability of other vendors providing the same or similar products and services at a comparable price. Possible outcomes include irrevocably broken trust (with no further relationship with this vendor), degraded trust (involving more cautious dealings with the vendor) or restored trust (wherein a continued relationship is maintained with the vendor)."

\section{INFORMATION QUALITY AND TRUST}

Another key antecedent to trust in FinTech tools is information quality (Beldad, De Jong, \& Steehouder, 2010). Information quality refers to a variety of concepts including error-freeness (Bart, Shankar, Sultan, \& Urban, 2005), accuracy, currency, and completeness of information (Kim, Song, Braynoy, \& Rao, 2005), along with correctness in spelling, grammar, and syntax (Koehn, 2003). The content quality (referring to the usefulness, accuracy, and completeness of the information offered) increases customers' trust in using an electronic service (Liao, Palvia, \& Lin, 2006). Information quality is considered even more critical in Robo-Advisors as the performance of the customers' portfolios rely on it. Epperson, Hedges, Singh, and Gabel (2015) echo these findings, suggesting that investment expertise is one of the critical factors when selecting a Robo-Advisor 


\section{Issues in Information Systems \\ Volume 19, Issue 1, pp. 128-138, 2018}

service. If content provided by Robo-Advisors is inaccurate, outdated, incomplete, misleading, and thereby useless, it will lead to degraded trust in the Robo-Advisor and its provider. Each new generation of Robo-Advisors offers features to better address information quality factors such as information completeness, accuracy, relevancy, and usefulness. However, even the third generation of Robo-Advisors currently on the market are not considered to be a major disruptive innovation due partly to the lack of expected information quality. Documented disappointment indicates that Robo-Advisors are mainly process automation rather than AI (artificial intelligence) or deep learning (Nanalyze, 2016).

Self-learning AI algorithms have just started to emerge and form a wave of fourth generation Robo-Advisors. EquBot launched its first retail Robo-Advisor using IBM's Watson AI technology in October 2017. It uses AI to choose stocks by ranking investment opportunities based on fundamentals such as profit growth and valuation with considerably better information processing capacity than humans (one million pieces of information a day including earnings releases, economic data, consumer trends, industry developments, and headlines news) (Lim, 2017). AI is, by definition, capable of machine learning, eliminating the necessity of human reprogramming. Financial advisors consider Robo-Advisors to be a suitable tool for managing smaller accounts due partially to the low cost involved. Customers with larger accounts are less concerned with the cost of advising than with flexibility. A more pressing reason would be, however, that Robo-Advisors have failed to demonstrate a superior level of performance in sizable portfolios. New generation Robo-Advisors with artificial intelligence algorithms might shift the horizon and help wealth managers respond more dynamically to customers' needs. As sophisticated algorithms become available, it will be possible to accommodate investment techniques like factor-based investing, which is rarely embedded in extant Robo-Advisors.

\section{INTERFACE QUALITY AND TRUST}

Interface quality is considered to be the main vehicle in achieving information transparency and consists of various factors including ease-of-use features, good graphical characteristics, and tailored user interface/services. A prior study illustrates that transparency through a consolidated user interface is an important criterion in selecting Robo-Advisors (Salo, 2017). With a transparent user interface, users can see an overview of their own investment portfolios without relying on the advisor's interpretation. Although it is partly related to information completeness, transparency can be achieved only through a welldesigned interface.

It is well documented that the perceived ease of using a particular technology is one of the key factors affecting trust. In fact, the perceived ease is a core construct in the technology acceptance model (Davis, 1989). Graphical characteristics are another key component in interface quality, augmenting the delivery of rich information. Kim and Moon (1998) found that an interface without proper graphical components such as a clipart degrades the trustworthiness of the service provider. The study illustrates that three-dimensional, dynamic clipart enhances the users' feelings of trust towards the banking system. Moreover, the color layout of the interface also plays a critical role in the perceived trustworthiness of online banking. Lastly, customizability, the ability to tailor products, services, and transactional environments to target users, is also positively related to trust (Koufaris \& Hampton-Sosa, 2004).

The current study focuses primarily on the design artifacts of Robo-Advisors although there are many other trust antecedents in the socio-cultural domain. For instance, experience and proficiency in Internet usage may play a critical role in developing trust in electronic services as evidenced by one study (Corbitt, Thanasankit, \& Yi, 2003). Echoing this, Reuba suggests that a higher impact from Robo-Advisors is expected as millennials acquire more money to invest (Reuba, 2017).

\section{METHODOLOGY}

A primary objective in the current study is the predictive capability of the dynamic Robo-Advisor trust model. For this study a design science approach was adopted for the following reasons. First of all, conventional social science research methodology is more suitable for investigating cause and effect relationships in controlled environments. The current study requires intervention and observation in a realistic Robo-Advisor market environment. The impact of different interventions on newly emerging technologies like Robo-Advisors under a variety of scenarios is rather difficult to analyze with a social science approach. Secondly, we aim to explore the implication of different Robo-Advisor implementation strategies in a contiguous time frame under a dynamically changing environment. Social science approaches are preferable in a cross-sectional study or longitudinal study where only a few discrete data collection points exist. Lastly, the complexity of the Robo-Advisor ecosystem 


\section{Issues in Information Systems \\ Volume 19, Issue 1, pp. 128-138, 2018}

and trust building mechanisms make it difficult to assess the implications of alternative design choices and strategies and to determine whether more appropriate approaches exist under the prevailing conditions. Complexity theory is suitable in addressing such characteristics of the problem domain and a framework is available for the design science approach. This approach mandates the utilization of an IT artifact that can be studied under a variety of conditions. Since the trust building mechanism in Robo-Advisors is dynamic in nature, the artifact proposed in this research is a model suite that will allow decision makers to assess the impact of alternative decisions regarding the implementation of Robo-Advisors under varying conditions.

Complexity theory represents the research framework adopted in the current study. It is the study of the phenomena which emerge from a collection of interacting objects (Johnson, 2007) and investigates the dynamics of interacting objects as well as the chronological evolution of a phenomenon (Choi, Nazareth, \& Ngo-Ye, 2017). A complex system consists of multiple actors who are interacting dynamically. Actors are autonomous since their decisions are independent based on each actor's strategies, prior experiences, accumulated knowledge, and the prevailing environmental conditions. The core characteristics of complexity theory is that a central planning or controlling mechanism is not embedded in the system. A complex system, which is not premeditated or planned, emerges often times, with unexpected results from the mixture of individual behaviors of the actors.

Design science approaches typically utilize modeling techniques. Although multiple modeling techniques are available for studying the adoption of technology innovations, including deterministic, stochastic, econometric, and simulation models (Choi, Nazareth, \& Ngo-Ye, 2017), an agent-based simulation technique is the most suitable for implementing a complexity theory framework because complexity theory is embedded in the technique. Agent-based modeling is a computational simulation approach that permits the study of phenomena over time (Bonabeau, 2002). Agents represent actors in the complexity theory framework. Agents are entities of the system that generates autonomous behaviors as actors do in complexity theory. Once the behavior of an individual agent is prescribed, the effectiveness of its logic is assessed by interacting operators (Ngo-Ye, Nazareth, \& Choi, 2017). Again, there is no prescribed or planned central controlling logic. The complex characteristics of the system are rendered by autonomous behavior and interactions among agents. This is counter to discrete event simulation, which utilizes predefined rules to control and govern the behavior of the system. An agent can represent market entities like customers, vendors, products, services, etc. The simulation model detailed in the current study adopts a complexity theory view and agent-based modeling techniques to offer a better understanding of the Robo-Advisor market by permitting interactions among multiple Robo-Advisor users and Robo-Advisor services as represented by agents. The modeling was conducted using NetLogo, currently the most popular agent-based simulation environment.

\section{MODEL DEVELOPMENT}

Construction and validation of the model are closely interwoven into an agent-based model. A systematic literature survey was done to form the basis for the initial identification of model components. The current study aims to report the completion of model component design and behavior coding. Parameter estimation and function development have already been completed. The next stage will see the calibration and testing of the model using a set of scenarios. The model was assembled in NetLogo, which is an effective agent-based simulation platform. The NetLogo platform enables researchers to construct the visual components of the simulation interface and supports the logo programming language to code agent behaviors. The user interface of the agent-based simulation model is presented in Figure 2.

This model depicts dynamic interactions between multiple Robo-Advisors and clients (potential customers of Robo-Advisors). Multiple Robo-Advisors are modeled to depict service switching cases. The numbers of available Robo-Advisors and clients are user-adjustable through sliders in the simulation interface, allowing decision makers to simulate the different conditions of the financial service industry where multiple Robo-Advisors are offered to many customers. The model assumes that the RoboAdvisor services are offered at a variety of price levels to accommodate the fact that Robo-Advisors' customers are primarily cost driven. Another factor affecting decision making is trust, which is considered to be a critical precondition for the adoption of electronic services (Beldad, De Jong, \& Steehouder, 2010). As stated in prior sections, the three dimensions affecting trust are considered here; information security, information quality, and interface quality. They are incorporated into the model, representing a key trust building mechanism. The model was developed using the Dynamic Security-Trust lifecycle model as a basis (Choi \& Nazareth, 2014) to capture the impact of security strategies on trust. To accommodate other dimensions like information quality and interface quality an extensive series of modifications, recalibrations, and extensions have been performed to ensure that the model truly reflects the Robo-Advisor market. 


\section{Issues in Information Systems \\ Volume 19, Issue 1, pp. 128-138, 2018}

Average attractiveness throughput is a user adjustable value representing the favorability of adopting Robo-Advisor services in general. It serves as a basis to generate an individual customer's psychological throughput. An individual Robo-Advisor's attractiveness is determined by the two critical factors affecting the choice of Robo-Advisors, namely, price and trustworthiness. If the Robo-Advisor's attractiveness exceeds the customer's psychological throughput (which is generated based on the average attractiveness throughput), the customer decides to adopt and use the specific Robo-Advisor provider. Information on price and trustworthiness is available to all potential Robo-Advisor customers in the model. Price is selected based on economic factors, which is a user-adjustable input variable and represents overall economic performance in an industry. Growing economies generate disparities between supply and demand, causing a price increase. So, individual prices for each Robo-Advisor are generated by the model at run time. Price affects the market share of each Robo-Advisor, and eventually, the competitive advantage over incumbent financial advisors.

Another factor affecting attractiveness is trustworthiness. It is determined by the combined level of security, information quality, and interface quality in the model, but these three factors only partially affect the initial trust level since the initial trust is mainly shaped by the customer's perceptions based on reputation and brand (Lynn, Van Der Werff, Hunt, \& Healy, 2016; Pearson \& Yee, 2013). A portion of ongoing trust fluctuates due to security- and privacy-related events in data transactions, data handling, data usage, and other factors related to security/privacy mismanagement. In the simulation interface, the average offense severity represents the average level of security breaches. Individual offense severity for a specific Robo-Advisor is selected at run time. In the event of a security breach, the trustworthiness of the Robo-Advisor degrades to some extent (determined by the severity of the offense). As long as overall trustworthiness is not degraded enough to push the attractiveness of the specific Robo-Advisor below a certain threshold, the customer continues to use the service. A reconciliation process to restore trust is readily available in the event of security breaches, but it is dependent on reconciliation effectiveness (which is determined by the average reconciliation effectiveness level). The second dimension, information quality, represents the eventual outcome quality of the Robo-Advisors' digital service. Therefore, investment performance relies on information quality. More sophisticated AI algorithms can accommodate advanced investment techniques like factor-based investing and the algorithms in general are becoming more flexible due to deep learning. In other words, as Robo-Advisors become more powerful and flexible, they approach and even surpass the capability of human financial advisors. Information quality can be evaluated only after a period of use and rarely contributes to initial trust. The simulated user interface accepts the level of information quality as a user input and employs it as a critical ongoing trust-forming factor. As previously discussed, the dimensions of information quality include information completeness, accuracy, relevancy, usefulness, and currency. The last dimension affecting trustworthiness is interface quality. Interface quality is regarded as the main vehicle for achieving information transparency. The factors affecting interface quality includes ease-of-use and good graphical characteristics, along with services and a user interface tailored to the user. Color and graphics can be the basis for initial trust, but most of interface quality, like ease-of-use features and customizability, contribute to ongoing trust. Interface quality is adjustable in a slider as a user input.

The model incorporates two breeds of agents, namely, Robo-Advisors and human clients. Desk-top shaped agents represent Robo-Advisors services while person shaped agents represent human clients. Red colored clients signify clients who are currently using a Robo-Advisor, while blue colored clients denote clients who are not currently using any Robo-Advisors. The graph located underneath sliders represent population of Robo-Advisors adopters. The simulation interface offers alternative options for enabling the reconciliation process and trust offenses (represent security breaches). They are located below the populations graph.

\section{SCENARIOS AND PROPOSITIONS}

The purpose of scenario analysis is twofold. Firstly, it is interwoven into the validation process. Calibrating the model and testing that it is functioning accurately are the integral parts of model validation. A realistic set of scenarios is needed for this procedure to validate that the model functions accurately and closely replicates the real world. Second, once the model is shown to behave satisfactorily, a series of analyses exploring specific scenarios or decision-making strategies can be performed to explain the outcomes. This will form the final stage of the current research stream.

The first group of scenarios (group A) is devised to explore the impact of offense severity and different forms of reconciliation tactics under a variety of economic conditions (depicted in Table 1). Once a security breach occurs, the Robo-Advisor's trustworthiness decreases. The eventual level of trustworthiness relies on the following two factors; the level to which it dropped depending on the severity of the security breach and how effective the corrective and remedial actions were (the effectiveness 


\section{Issues in Information Systems \\ Volume 19, Issue 1, pp. 128-138, 2018}

of reconciliation tactics). However, there is one more dimension with which the attractiveness of the Robo-Advisor is determined other than trustworthiness, namely, price. Hence, the first group of scenarios need to be investigated under each level of economic conditions. Economic conditions and the resulting price points are more likely to shape the impact of offense severity then the choice of reconciliation tactics and thus form the basis for the first proposition.

Proposition 1: Economic conditions will significantly affect the role of offense severity and the choice of reconciliation tactics, and thereby shape the ongoing trust maintenance process.
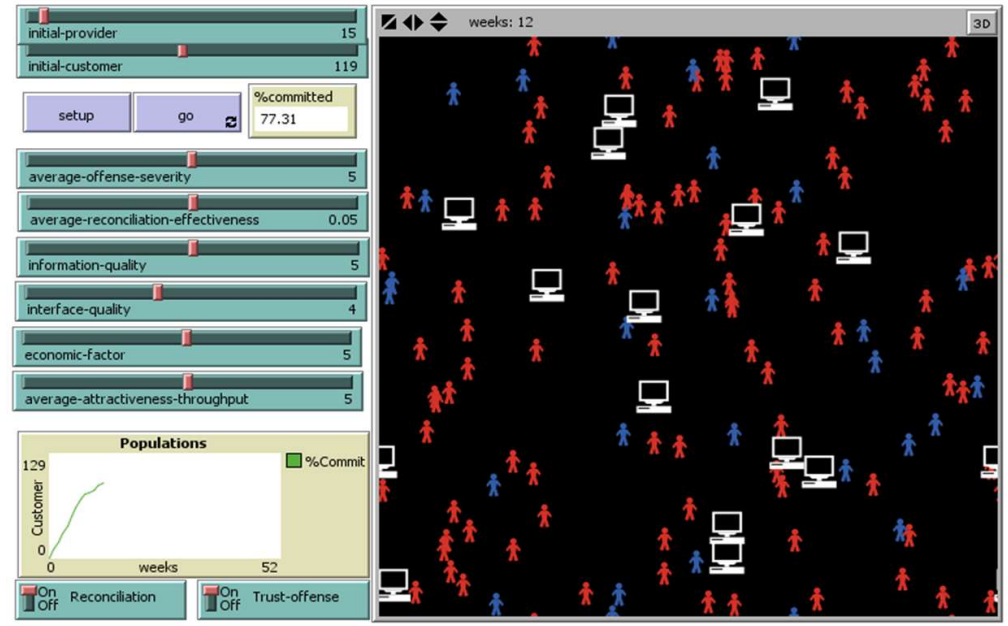

Figure 2. The Dynamic Trust Model for Robo-Advisors

Table 1. Scenarios Group A to Contrast Economic Conditions

\begin{tabular}{|l|l|l|l|}
\hline Scenario & Offense Severity & Reconciliation Tactics & Economic Conditions \\
\hline A1,2,3 & Low & Low & Low, Medium, High \\
\hline A4,5,6 & Low & Moderate & Low, Medium, High \\
\hline A7,8,9 & Low & High & Low, Medium, High \\
\hline A10,11,12 & Moderate & Low & Low, Medium, High \\
\hline A13,14,15 & Moderate & Moderate & Low, Medium, High \\
\hline A16,17,18 & Moderate & High & Low, Medium, High \\
\hline A19,20,21 & High & Low & Low, Medium, High \\
\hline A22,23,24 & High & Moderate & Low, Medium, High \\
\hline A25,26,27 & High & High & Low, Medium, High \\
\hline
\end{tabular}

Again, the second group of scenarios (group B) aims to explore the impact of offense severity and different forms of reconciliation tactics. However, the implication of information quality is the main dimension of interest this time (the group B of scenarios is illustrated in Table 2). As previously discussed, the information quality level is a critical factor affecting the trust forming process and certainly plays a key role in the ongoing trust lifecycle. The current study investigates the role of information quality in the event of an emergency situation where the maintenance of trust becomes necessary. The level of information quality is more likely to affect the impact of the offense severity than the choice of reconciliation tactics. Therefore, the second proposition is formed as follows.

Proposition 2: Information quality will significantly affect the ongoing trust maintenance process. 
Issues in Information Systems

Volume 19, Issue 1, pp. 128-138, 2018

Table 2. Scenarios Group B to Contrast Information Quality

\begin{tabular}{|l|l|l|l|}
\hline Scenario & Offense Severity & Reconciliation Tactics & Information Quality \\
\hline B1,2,3 & Low & Low & Low, Medium, High \\
\hline B4,5,6 & Low & Moderate & Low, Medium, High \\
\hline B7,8,9 & Low & High & Low, Medium, High \\
\hline B10,11,12 & Moderate & Low & Low, Medium, High \\
\hline B13,14,15 & Moderate & Moderate & Low, Medium, High \\
\hline B16,17,18 & Moderate & High & Low, Medium, High \\
\hline B19,20,21 & High & Low & Low, Medium, High \\
\hline B22,23,24 & High & Moderate & Low, Medium, High \\
\hline B25,26,27 & High & High & Low, Medium, High \\
\hline
\end{tabular}

Finally, the third group of scenarios (group C) is listed below in Table 3, offering a tool to investigate the impact of offense severity and the types of reconciliation tactics for the different levels of interface quality. This is a logical next step since interface quality has been identified as a core dimension in building trust. Therefore, the third proposition is as follows.

Proposition 3: Interface quality will play a key role in the ongoing trust maintenance process.

Table 3. Scenarios Group C to Contrast Interface Quality

\begin{tabular}{|l|l|l|l|}
\hline Scenario & Offense Severity & Reconciliation Tactics & Interface Quality \\
\hline C1,2,3 & Low & Low & Low, Medium, High \\
\hline C4,5,6 & Low & Moderate & Low, Medium, High \\
\hline C7,8,9 & Low & High & Low, Medium, High \\
\hline C10,11,12 & Moderate & Low & Low, Medium, High \\
\hline C13,14,15 & Moderate & Moderate & Low, Medium, High \\
\hline C16,17,18 & Moderate & High & Low, Medium, High \\
\hline C19,20,21 & High & Low & Low, Medium, High \\
\hline C22,23,24 & High & Moderate & Low, Medium, High \\
\hline C25,26,27 & High & High & Low, Medium, High \\
\hline
\end{tabular}

\section{FUTURE PLANS AND CONCLUDING REMARKS}

This research examines the role of trust in the implementation of Robo-Advisors, focusing on the process of trust violation and restoration along three dimensions, namely, information security, information quality, and interface quality. The second phase of the ongoing study is reported here, involving comprehensive theoretical underpinnings and a literature survey to develop a realistic agent-based simulation model. It devises the interface of the dynamic trust model for Robo-Advisors, reflecting the ecosystem of Robo-Advisors. Agents' behaviors have been coded using the Netlogo platform based on the documented studies and reports. A complete set of scenarios were developed for the purpose of model validation, the evaluation of alternative strategies, and the assessment of environmental conditions. These steps and processes will be detailed in the final installment of the study. Simulation outcomes will be analyzed to see if the model behaves reasonably and follows historical trends. Then the implications of alternative strategies and conditions will be explored with a set of multiple simulation runs. The insights gained through the analysis of the simulation outcomes should prove priceless to financial advisors when evaluating RoboAdvisors' implementation and adoption decisions. The potential contributions of this research include a better understanding of the ongoing trust lifecycle in the onset of trust degradation events in information security, information quality, and interface quality. 


\section{REFERENCES}

Aiken, K. D., \& Bousch, D. M. (2006). Trustmarks, objective-source ratings, and implied investments in advertising: Investigating online trust and the context-specific nature of internet signals. Journal of the Academy of Marketing Science, 34, 308-323.

Bart, Y., Shankar, V., Sultan, F., \& Urban, G. L. (2005). Are the drivers and role of online trust the same for all web sites and consumers? A large-scale exploratory empirical study. Journal of Marketing, 69, 133-152.

Beldad, A., De Jong, M., \& Steehouder, M. (2010). How shall I trust the faceless and the intangible? A literature review on the antecedents of online trust. Computers in Human Behavior, 26, 857-869.

Bigley, G., \& Pearce, J. (1998). Straining for shared meaning in organization science: Problems of trust and distrust. Academy of Management Review, 23(3), 405-421.

Bonabeau, E. (2002). Agent-Based Modeling: Methods and Techniques for Simulating Human Systems. 99(3), $7280-7287$.

Chellappa, R., \& Pavlou, P. (2002). Perceived information security, financial liability and consumer trust in electronic commerce transactions. Logistics Information Management, 15(5/6), 358-368.

Choi, J., \& Nazareth, D. L. (2014). Repairing trust in an e-commerce and security context: an agent-based modeling approach. Information Management and Computer Security, 22(5), 490-512.

Choi, J., Nazareth, D. L., \& Ngo-Ye, T. L. (2017). The Effect of Innovation Characteristics on Cloud Computing Diffusion. Journal of Computer Information Systems. doi:10.1080/08874417.2016.1261377

Corbitt, B. J., Thanasankit, T., \& Yi, H. (2003). Trust and e-commerce: A study of consumer perceptions. Electronic Commerce Research and Applications, 2, 203-215.

Davis, F. (1989). Perceived usefulness, perceived ease of use, and user acceptance of information technology. MIS Quarterly, 13(3), 319-340.

Epperson, T., Hedges, B., Singh, U., \& Gabel, M. (2015). Hype vs. Reality: The Coming Waves of "Robo" Adoption, Insights from the A. T. Kearney 2015 Robo-Advisory Services Study. A. T. Kearney.

Flavian, G., \& Guinaliu, M. (2006). Consumer trust, perceived security and privacy policy: three basic elements of loyalty to a web site. Industrial Management and Data Systems, 106(5), 601-620.

Gold, N. A., \& Kursh, S. R. (2017). Counterrevolutionaries in the Financial Service Industry: Teaching Disruption-A Case Study of RoboAdvisors and Incumbent Responses. Business Education Innovation Journal, 9(1), 139-146.

Gregor, S., \& Hevner, A. R. (2013). Positioning and Presenting Design Science Research for Maximum Impact. MIS Quarterly, $37(2), 337-355$.

Hevner, A. R., March, S. T., Park, J., \& Ram, S. (2004). Design Science in Information Systems Research. MIS Quarterly, 28(1), 75-105.

Holmes, J. (1991). Trust and the appraisal process in close relationships. In W. Joens, \& D. Perlamn (Eds.), Advances in Personal Relationships. London: Jessica Kingsley.

Johnson, N. (2007). Simply Complexity: a clear guide to complexity theory. Oxford, England: Oneworld Publications.

Kim, D. J., Song, Y. I., Braynoy, S. B., \& Rao, H. R. (2005). A multidimensional trust formation model in B-to-C e-commerce: A conceptual framework and content analyses of academia/practitioner perspectives. Decision Support Systems, 40, 143-165. 
Kim, J., \& Moon, J. (1998). Designing towards emotional usability in customer interfaces: Trustworthiness of cyber-banking system interfaces. Interacting with Computers, 10, 1-29.

Koehn, D. (2003). The nature of and conditions for online trust. Journal of Business Ethics, 43, 3-19.

Koufaris, M., \& Hampton-Sosa, W. (2004). The development of initial trust in an online company by new customers. Information \& Management, 41, 377-397.

Lee, S., Choi, J., Ngo-Ye, T. L., \& Cummings, M. (2018). Modeling Trust in the Adoption Decision Process of Robo-Advisors: An Agent-Based Simulation Approach. Proceedings of the Tenth Midwest Association for Information Systems Conference. Saint Louis, Missouri: Association for Information Systems.

Lewicki, R. J., \& Tomlinson, E. C. (2003, December). Trust and Trust Building. Retrieved May 4, 2018, from Beyond Intractability: https://www.beyondintractability.org/essay/trust_building

Lewicki, R., \& Bunker, B. (1995). Trust in Relationships: A Model of Trust Development and Decline. In M. Deutsch, B. Bunker, \& J. Rubin, Conflict, Cooperation and Justice (pp. 133-173). San Francisco: Jossey-Bass.

Lewicki, R., \& Bunker, B. (1996). Developing and maintaining trust in working relationships. In R. Kramer, \& T. Tyler (Eds.), Trust in Organizations: Frontier of Theory and Research (pp. 114-139). Thousand Oaks, CA: Sage.

Liao, C., Palvia, P., \& Lin, H. (2006). The roles of habit and website quality in e-commerce. International Journal of Information Management, 26, 469-483.

Lim, P. (2017, October 25). The First Ever Fund Managed by a Robot is Here: So Far It's Beating the Market. Retrieved May 3, 2018, from Time: http://time.com/money/4993744/robot-mutual-fund-beating-stock-market/

Lynn, T., Van Der Werff, L., Hunt, G., \& Healy, P. (2016). Development of a Cloud Trust Label: A Delphi Approach. Journal of Computer Information Systems, 56(3), 185-193.

McKnight, D., Choudhury, V., \& Kacmar, C. (2002). Developing and validating trust measures for e-commerce: an integrative typology. Information Systems Research, 13(3), 334-359.

Moulliet, D., Stolzenbach, J., Majonek, A., \& Völker, T. (2016, August). The Expansion of Robo-Advisory in Wealth Management. Retrieved May 3, 2018, from Deloitte: https://www2.deloitte.com/content/dam/Deloitte/de/Documents/financial-services/Deloitte-Robo-safe.pdf

Nanalyze. (2016, January 29). The Real Truth Behind The Rise of Robo-Advisors. Retrieved May 3, 2018, from Nanalyze: https://www.nanalyze.com/2016/01/the-real-truth-behind-the-rise-of-robo-advisors/

Ngo-Ye, T. L., Nazareth, D. L., \& Choi, J. (2017). Trust in Cloud Computing: Maintaining Long-Term Relationships. Journal of Information Technology Management, 28(3), 25-38. Retrieved May 3, 2018, from http://jitm.ubalt.edu/XXVIII3/article2.pdf

Pearson, S., \& Yee, G. (2013). Privacy and Security for Cloud Computing: Computer Communications and Networks. London, UK: Springer-Verlag.

Peffers, K., Tuunanen, T., Rothenberger, M. A., \& Chatterjee, S. (2008). A Design Science Research Methodology for Information Systems Research. Journal of Management Information Systems, 24(3), 45-77.

Ray, S., Ow, T., \& Kim, S. (2011). Security assurance: how online service providers can influence security control perceptions and gain trust. Decision Sciences Journal, 42(2), 391-412. 


\section{Issues in Information Systems}

Volume 19, Issue 1, pp. 128-138, 2018

Reichheld, F. F., \& Schefter, P. (2000). E-Loyalty: Your Secret Weapon on the Web. Harvard Business Review, 78(4), 105114.

Reuba, K. (2017, December 5). Robo-Advisors: Early Disruptors in Private Wealth Management. Retrieved May 3, 2018, from Allianz Investment Themes and Strategy: https:/www.allianzgi.com/home/insights/artificial-intelligence/roboadvisors-early-disruptors-in-private-wealth-management

Roca, J. C., Carcia, J. J., \& De La Vega, J. J. (2009). The importance of perceived trust, security and privacy in online trading systems. Information Management and Computer Security, 17(2), 96-113.

Salo, A. (2017). Robo Advisor, Your Reliable Partner? Building a Trustworthy Digital Investment Management Service. Master's Thesis, University of Tampere, School of Management, Tampere, Finland.

Suh, B., \& Han, I. (2003). The impact of customer trust and perception of security control on the acceptance of electronic commerce. International Journal of Electronic Commerce, 7(3), 135-161. 